\title{
Distant metastasis of follicular thyroid carcinoma to the mandible: a rare case report
}

\author{
Young-Tae Jeon, Chul-Hwan Kim, Sung-Min Park, Min-Kyoo Kim \\ Department of Oral and Maxillofacial Surgery, College of Dentistry, Dankook University, Cheonan, Korea
}

\begin{abstract}
J Korean Assoc Oral Maxillofac Surg 2019;45:294-298)
Treatment of metastatic carcinoma of the oral cavity can be a challenge due to its rarity. The current case report details metastatic carcinoma of the mandible originating from follicular thyroid carcinoma, which is the second most prevalent tumor of the thyroid. As the mandibular lesion developed, the primary thyroid lesion of thyroid. Here, we report a case of metastatic carcinoma of the mandible in a 67-year-old female that was treated with partial mandibulectomy and fibular free flap.
\end{abstract}

Key words: Neoplasm metastasis, Mandible, Thyroid neoplasms, Adenocarcinoma, Follicular

[paper submitted 2019. 2. 11 / revised 2019. 3. 19 / accepted 2019. 3. 27]

\section{Introduction}

Due to its relative rarity, metastatic carcinoma in the oral region can be difficult to treat. Metastatic carcinoma accounts for approximately $1 \%$ of cancer in the oral region ${ }^{1}$. The most common region of primary tumors leading to mandibular metastasis is the lung in men and breast in women ${ }^{2}$. Metastasis of tumors to the jaw bone can be especially difficult to detect because they commonly appear as an osteolytic lesion, which can be difficult to discriminate from odontogenic cysts. According to the literature, in $30 \%$ of cases with metastasis to the jaw, the primary lesion was asymptomatic and was not detected before the metastatic lesion ${ }^{3}$.

This report presents a case of oral carcinoma metastasized from follicular thyroid carcinoma. Follicular cell cancers are the second most common thyroid cancer after papillary thyroid carcinoma. These tumors are derived from follicular cells of the thyroid region and are more likely to be found

\section{Chul-Hwan Kim}

Department of Oral and Maxillofacial Surgery, College of Dentistry, Dankook University, 119 Dandae-ro, Dongnam-gu, Cheonan 31116, Korea TEL: +82-41-550-0271 FAX: +82-41-551-8988

E-mail:kimchoms@dankook.ac.kr

ORCID: https://orcid.org/0000-0002-5199-2420

(c) This is an open-access article distributed under the terms of the Creative Commons Attribution Non-Commercial License (http://creativecommons.org/ licenses/by-nc/4.0/), which permits unrestricted non-commercial use, distribution, and reproduction in any medium, provided the original work is properly cited.

Copyright (C) 2019 The Korean Association of Oral and Maxillofacial Surgeons. All rights reserved. among females 40 to 60 years old. They account for $10 \%$ of all thyroid malignancies ${ }^{4}$. They are usually asymptomatic and are initially detected via palpation of a solid nodule, multinodular goiter, or cervical lymphadenopathy ${ }^{5}$. In this report, we describe a case of follicular thyroid carcinoma in which metastasis to the mandibular anterior-posterior region was detected prior to that of the primary thyroid carcinoma.

\section{Case Report}

In October 2017, a 67-year-old female was referred to the Department of Oral and Maxillofacial Surgery of Dankook University Dental Hospital (Cheonan, Korea) for evaluation of gingival and facial swelling. Prior to the patient's first visit to our hospital, the swelling had been attributed to follicular carcinoma originating from the thyroid region via fine needle aspiration performed at a local hospital for mandibular asymmetry evaluation. The patient was a non-smoker and suffered from hypertension; she had no family history of thyroid disease. The mandibular lesion had been detected approximately 5 months prior in a local dental clinic, and the patient was recommended to visit a higher-tier hospital for further evaluation of a suspected mandibular cyst. However, no further visits to the hospital were made before September, when the patient started to suffer from facial swelling and paresthesia of the right mandibular region. At that time, she visited another local hospital, where fine needle aspiration biopsy was 
performed. The patient was finally referred to our hospital for further evaluation and treatment. Gingival swelling of the right mandibular area was observed upon initial intra-oral examination, and extra-oral examination revealed facial asymmetry with swelling and paresthesia of the right mandibular area.(Fig. 1)

$\mathrm{X}$-ray radiographs (panoramic radiograph, computed tomography $[\mathrm{CT}]$, and magnetic resonance imaging [MRI]) were collected for further diagnosis.(Fig. 2) Axial CT and sagittal MRI images revealed a large ( $52 \mathrm{~mm} \times 44 \mathrm{~mm} \times 34$ $\mathrm{mm}$ ) mass with a lobulated margin in the right mandibular body. An identical mass in the corresponding region was observed on panoramic view. There was no evidence of a lesion effect one the adjacent teeth - such as resorption of roots or occlusion change. A whole-body bone scan and positron emission tomography-CT (PET-CT) were performed for further analysis (Fig. 3), revealing a mass in the same region and additional identification of the primary lesion in the thyroid
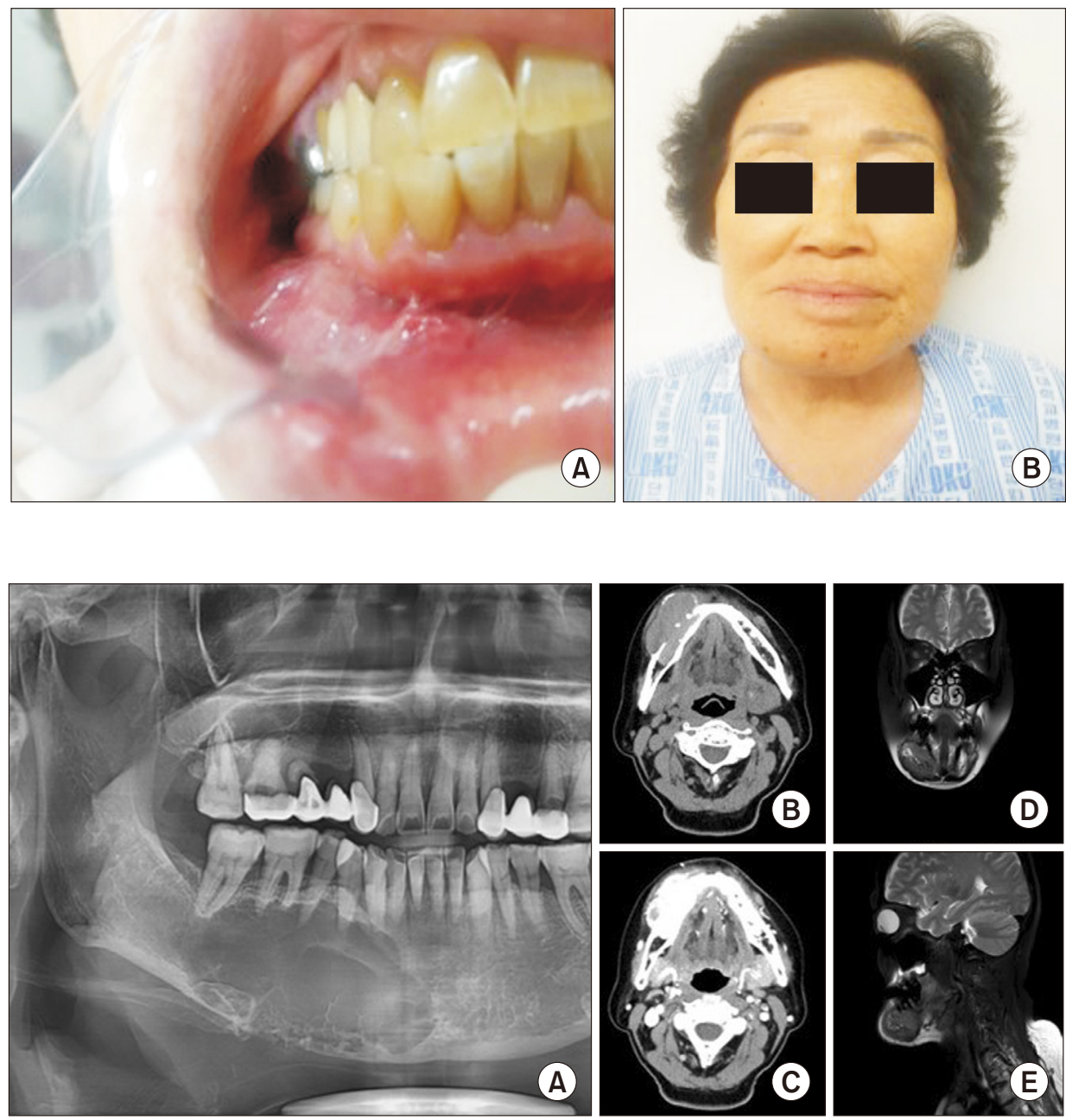

Fig. 2. A. Panoramic radiograph shows a lobulated or scalloped margin mass involving the right mandible. Absorption of the roots are not apparent. B. Computed tomography (CT) without enhancement shows bony destruction in same area. C. CT with enhancement reveals enhancing mass involving in-
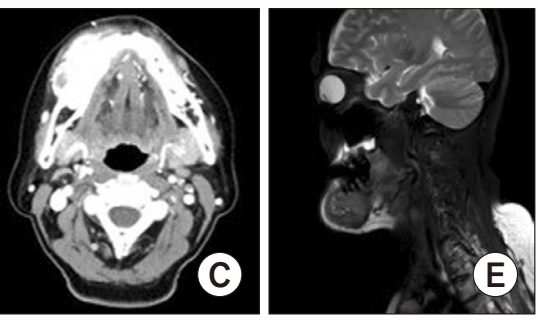
ner and outer cortex of the right mandible body. It is different with any other odontogenic cysts. D, E. Magnetic resonance imaging presents enhancing mass involving the right mandible body same as computed tomography.

Young-Tae Jeon et al: Distant metastasis of follicular thyroid carcinoma to the mandible: a rare case report. J Korean Assoc Oral Maxillofac Surg 2019
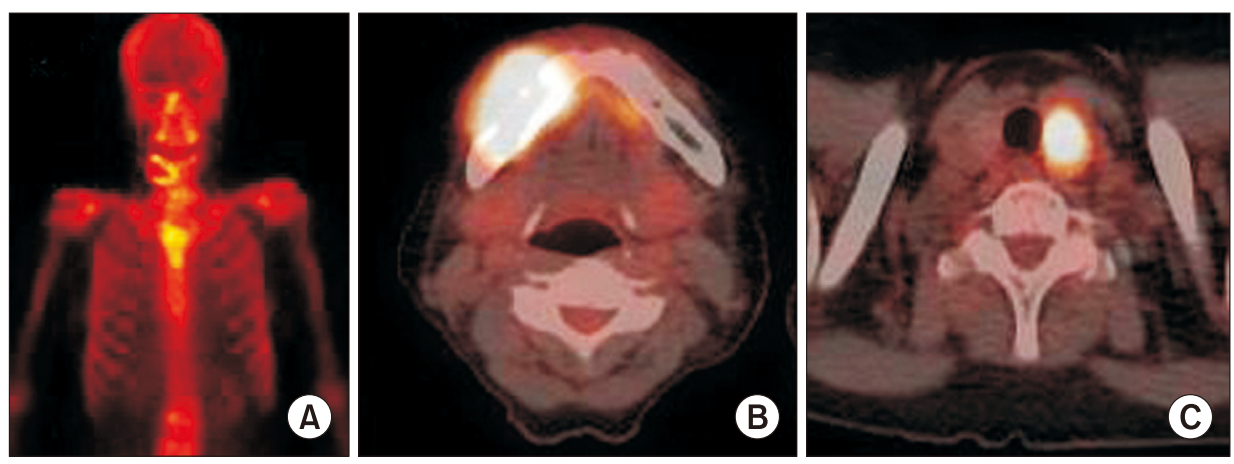

Fig. 3. A. Bone scan shows swelling with hollow mass in right mandible. B, C. Positron emission tomography-computed tomography reveals hypermetabolic lesion in right mandible body and left thyroid nodule.

Young-Tae Jeon et al: Distant metastasis of follicular thyroid carcinoma to the mandible: a rare case report. J Korean Assoc Oral Maxillofac Surg 2019 

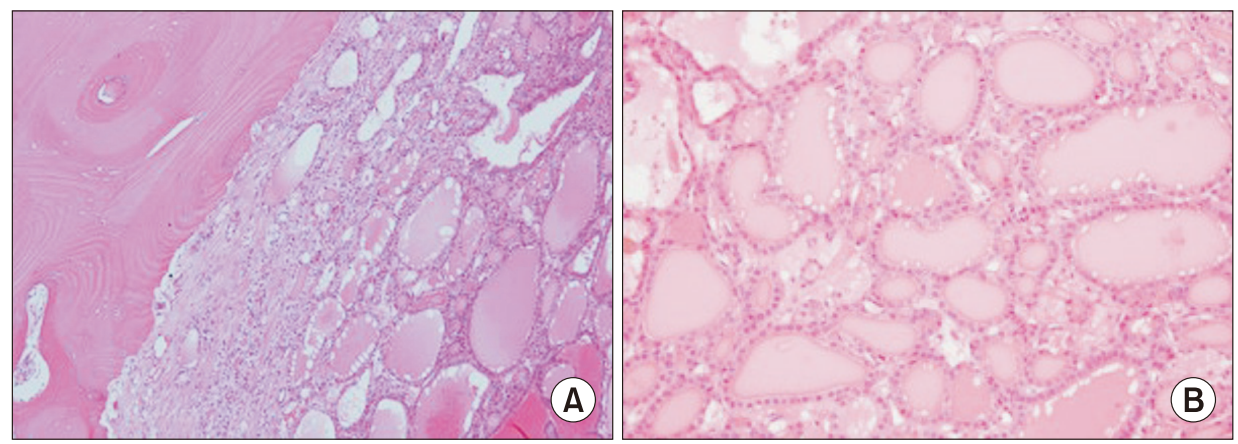

Fig. 4. A. Microscopic characteristics. A. Cluster of colloid-filled follicles are identified with matured bone tissue of mandible ( $\mathrm{H} \& \mathrm{E}$ staining, $\times 100)$. $B$. Neoplastic follicles are surrounded with tumor cells (H\&E staining, $\times 200$ ). Young-Tae Jeon et al: Distant metastasis of follicular thyroid carcinoma to the mandible: a rare case report. J Korean Assoc Oral Maxillofac Surg 2019
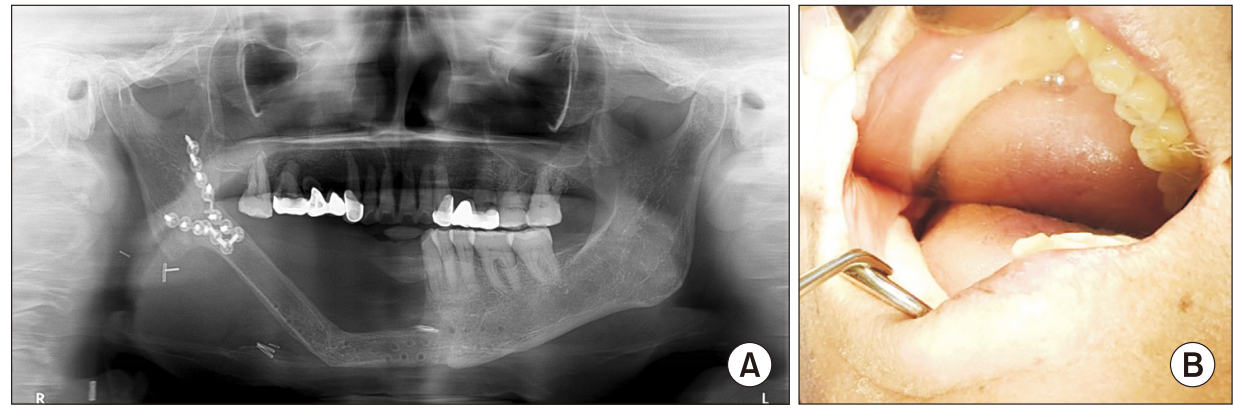

Fig. 5. Current state of the patient. A. Postoperative panoramic view was taken in March 2018. Plate and screws of anterior mandible was removed. B. Osteocutaneous fibular free flap is stabilized and keratinized. Temporary denture is used for adaptation.

Young-Tae Jeon et al: Distant metastasis of follicular thyroid carcinoma to the mandible: a rare case report. J Korean Assoc Oral Maxillofac Surg 2019 area. The primary thyroid lesion was referred to the ENT (ear, nose and throat) department for further management, and additional fine needle aspiration of this lesion was performed for additional diagnosis, revealing follicular thyroid carcinoma.

Partial mandibulectomy from the right mandibular angle to the left mandibular central incisor and supraomohyoid neck dissection were performed. A fibular free flap was used to restore mandibular stability and function. Results of the excisional biopsy revealed that the lesion was metastatic carcinoma originating from follicular thyroid carcinoma of the thyroid gland.(Fig. 4) Total thyroidectomy was performed in the ENT department one month later. The patient received subsequent radioactive iodine therapy in March 2018, and no specific signs were observed on follow-up radiographic images. The patient is currently using a temporary denture, and the mandibular operation field remains in a stabilized state. (Fig. 5)

\section{Discussion}

Distant metastasis of thyroid carcinoma is rare, with bone (vertebrae, pelvis, and ribs) and lung as its most common metastatic sites ${ }^{6}$. Distant metastasis to the oral region from thyroid carcinoma accounts for approximately $3 \%$ of all oral metastatic carcinomas ${ }^{7}$. The vast majority of metastatic tumors of the jaw is clinically presents with intraoral or extraoral swelling accompanied by chin paresthesia and pain ${ }^{6,8}$. Although thyroid carcinomas in general affect women more often than men, equal incidence between the sexes has been found for metastasis ${ }^{9}$. The mandible is more commonly affected than the maxilla, with the posterior region being the most frequent site of metastasis ${ }^{2}$. Because follicular thyroid carcinomas are more prone to hematogenous spread in advanced forms, the posterior part of the mandible, located directly along the main blood stream of the inferior alveolar vessel, can be easily affected ${ }^{8,9}$. Furthermore, the vast amount of hematopoietic bone marrow situated in this particular region allows for much greater hematopoietic activity of this region compared to other mandibular areas. Thus, the posterior mandible can serve as a favorable niche for attracting metastatic tumor cells ${ }^{8,9}$. Moreover, not only does this type of carcinoma spread through a hematogenous route, but it also has a tendency to grow to an extremely vascularized form of tumor in its advanced stages. This high vascularity with pulsation may lead to incorrect diagnosis, such as arteriovenous malformation ${ }^{10}$. Therefore, consideration of bleeding control is necessary. The literature has recommended preoperative angiography and selective embolization or external carotid artery ligation for intraoperative bleeding control ${ }^{9,11}$. However, because thyroid carcinomas are more likely to spread hematogenously, their propensity for lymphatic spread is 
relatively small ${ }^{9}$. Neck dissection may not be necessary for cases without lymphadenopathy. Selective excision of suspicious lymph nodes along the internal jugular vein and frozen sectioning may be considered for "staging" and determination of the necessity of neck dissection ${ }^{9}$. For the case herein, no sign of tumors was found among the lymph node groups of the supraomohyoid region.

Complete treatment of metastatic carcinoma requires addressing both the primary lesion and metastatic site. Treatment for the primary thyroid lesion often involves high-dose radioiodine therapy or thyroidectomy, as it did in this case of follicular thyroid carcinoma. However, for cases with bone metastasis, sufficient concentration of radioiodine cannot be easily achieved via intravenous administration, and external beam radiation therapy can be considered as an alternative ${ }^{12}$. Most metastatic carcinoma cases are dealt with via surgical resection, radiation therapy, or a combination. Treatment of our patient was carried out in a similar way via partial surgical resection of the mandible and reconstruction with an osteocutaneous fibular free flap. A previous study reported a similar case of mandibular metastasis of papillary thyroid carcinoma treated through surgical resection and bone-impacted fibular free flap reconstruction, which was followed up for 17 months ${ }^{13}$.

The prognosis and survival rate of follicular thyroid carcinoma have been reviewed in several studies. The prognosis of follicular thyroid carcinoma depends on age, size, and extent of vascular invasion and distant metastasis. An overall 5 -year survival rate of $40 \%$ and 10 -year survival rate of $27 \%$ for bone metastasis of differentiated thyroid carcinoma have been reported ${ }^{14,15}$. More recently, in a systematic review literature of 59 cases of thyroid cancer with facial skeleton metastasis, the overall survival rate was $96 \%$ at 2 years and $59 \%$ at 5 years. Among them, 22 cases were treated with surgery and postoperative radioactive iodine, yielding a survival rate of $100 \%$ at 2 years and $71 \%$ at 5 years ${ }^{16}$.

Because of their rarity, metastatic follicular thyroid carcinoma of the mandible has an unknown exact incidence, and treatment of this type of carcinoma can be a clinical challenge. Early diagnosis and adequate surgical and radiological treatment are vital to long-term survival of patients suffering from this type of carcinoma. It is therefore recommended that the maxillofacial surgeon collaborate with relevant specialists for successful treatment of metastatic follicular thyroid carcinoma.

\section{ORCID}

Young-Tae Jeon, https://orcid.org/0000-0002-6448-1670

Chul-Hwan Kim, https://orcid.org/0000-0002-5199-2420

Sung-Min Park, https://orcid.org/0000-0001-8573-9071

Min-Kyoo Kim, https://orcid.org/0000-0003-2110-6819

\section{Authors' Contributions}

Y.T.J. participated in data collection and wrote the manuscript. C.H.K., S.M.P., and M.K.K. participated in the design and performed the draft. C.H.K. participated in the coordination and helped to draft the manuscript.

\section{Consent for Publishing Photographs}

Written informed consent was obtained from the patients for publication of this article and accompanying images.

\section{Conflict of Interest}

No potential conflict of interest relevant to this article was reported.

\section{References}

1. Meyer I, Shklar G. Malignant tumors metastatic to mouth and jaws. Oral Surg Oral Med Oral Pathol 1965;20:350-62.

2. Hirshberg A, Buchner A. Metastatic tumours to the oral region. An overview. Eur J Cancer B Oral Oncol 1995;31B:355-60.

3. Bodner L, Geffen DB. [Metastatic tumor of the jaw--diagnosis and management]. Refuat Hapeh Vehashinayim (1993) 2003;20:59-61, 81. Hebrew.

4. D'Avanzo A, Treseler P, Ituarte PH, Wong M, Streja L, Greenspan FS, et al. Follicular thyroid carcinoma: histology and prognosis. Cancer 2004;100:1123-9.

5. Sreedharan S, Pang CE, Chan GS, Soo KC, Lim DT. Follicular thyroid carcinoma presenting as axial skeletal metastases. Singapore Med J 2007;48:640-4.

6. Pittas AG, Adler M, Fazzari M, Tickoo S, Rosai J, Larson SM, et al. Bone metastases from thyroid carcinoma: clinical characteristics and prognostic variables in one hundred forty-six patients. Thyroid 2000;10:261-8.

7. Hirshberg A, Shnaiderman-Shapiro A, Kaplan I, Berger R. Metastatic tumours to the oral cavity - pathogenesis and analysis of 673 cases. Oral Oncol 2008;44:743-52.

8. Hirshberg A, Berger R, Allon I, Kaplan I. Metastatic tumors to the jaws and mouth. Head Neck Pathol 2014;8:463-74.

9. Vural E, Hanna E. Metastatic follicular thyroid carcinoma to the mandible: a case report and review of the literature. Am J Otolaryngol 1998;19:198-202.

10. Tovi F, Leiberman A, Hirsch M. Uncommon clinical manifestations in a case of thyroid carcinoma. Head Neck Surg 1984;6:9747.

11. Kearns DB, Robinson LD, Wright GL, Wickersham JK, Parke RB Jr. Skull metastases from follicular thyroid carcinoma. Arch Otolar- 
yngol Head Neck Surg 1988;114:454-6.

12. McHenry CR, Phitayakorn R. Follicular adenoma and carcinoma of the thyroid gland. Oncologist 2011;16:585-93.

13. Germain MA, Marandas P, Leridant AM, Domenge C, Julieron $\mathrm{M}$, Schlumberger M, et al. [Isolated mandibular metastasis of cancer of the thyroid. Mandibulectomy and reconstruction using a free vascularized peroneal graft]. Rev Stomatol Chir Maxillofac 1998;98:371-4. French.

14. Brennan MD, Bergstralh EJ, van Heerden JA, McConahey WM. Follicular thyroid cancer treated at the Mayo Clinic, 1946 through 1970: initial manifestations, pathologic findings, therapy, and outcome. Mayo Clin Proc 1991;66:11-22.

15. Schlumberger M, Tubiana M, De Vathaire F, Hill C, Gardet P, Travagli JP, et al. Long-term results of treatment of 283 patients with lung and bone metastases from differentiated thyroid carcinoma. J Clin Endocrinol Metab 1986;63:960-7.

16. Varadarajan VV, Pace EK, Patel V, Sawhney R, Amdur RJ, Dziegielewski PT. Follicular thyroid carcinoma metastasis to the facial skeleton: a systematic review. BMC Cancer 2017;17:225.

How to cite this article: Jeon YT, Kim CH, Park SM, Kim MK. Distant metastasis of follicular thyroid carcinoma to the mandible: a rare case report. J Korean Assoc Oral Maxillofac Surg 2019;45:294-298. https://doi.org/10.5125/jkaoms.2019.45.5.294 